\title{
Embriologia do siri Arenaeus cribrarius (Lamarck) (Crustacea, Brachyura, Portunidae) $^{1}$
}

\author{
Marcelo A.A. Pinheiro ${ }^{2}$ \\ Gustavo Yomar Hattori ${ }^{2}$
}

\begin{abstract}
Embryology of the swimming crab Arenaeus cribrarius (Lamarck) (Crustacea, Brachyura, Portunidae). Ovigerous females of Arenaeus cribrarius were collected at Ubatuba, São Paulo, and maintaining in aquaria under controlled temperature $\left(25 \pm 1^{\circ} \mathrm{C}\right)$, salinity $(35 \pm 1 \%$ ) and photoperiod (12:12). A small sample of eggs was collected for each $24 \mathrm{~h}$, for morphological description, chromatic changes and biometric analysis throughout the embryonic development. Eight egg stages were photographed and had their small and large diameters measured under a microscope provided with camera lucida. The egg's shape was low elliptical during all the embryogenesis and showed size increase $(24.4 \%)$ and volume $(91.1 \%)$, when the last stage were compared with the first one. According to mean egg diameter and volume, the eggs should be pooled in three different groups $(1-3 ; 4-6 ; 7-8)$ corresponding to initial, intermediate and final stages. The attained results were compared with the literature which emphasizing the species of Portunidae's family.
\end{abstract}

KEY WORDS. Crustacea, Brachyura, Portunidae, swimming crab, embryology

Arenaeus cribrarius (Lamarck, 1818) é um portunídeo que ocorre em águas costeiras rasas, principalmente na zona de arrebentação das ondas, onde se enterra no sedimento. Dentre as contribuições sobre sua biologia reprodutiva destacam-se: a descrição completa de seus estágios larvais (STUCK \& TRUESDALE 1988); maturidade sexual (PINHEIRO \& FRANSOZO 1998); comportamento reprodutivo em cativeiro (PINHEIRO \& FRANSOZO 1999); análise de sua fecundidade e potencial reprodutivo (PINHEIRo \& TERCEIRo 2000); e dinâmica reprodutiva (PINHEIRO \& FRANSOZO 2002).

A embriologia de crustáceos decápodos é um assunto escasso na literatura (ANDERSON 1982). Um dos trabalhos pioneiros foi de BOOLOOTIAN et al. (1959) que propuseram a classificação dos ovos dos crustáceos decápodos em dez estágios, com base na proporção vitelo/embrião e na origem/formação de estruturas internas peculiares. Outros autores têm considerado um número menor de estágios embrionários, variando de três a seis, sendo o agrupamento realizado, principalmente, com base na coloração e quantidade de vitelo (VALDES et al. 1991; SAINTE-MARIE 1993; PINHEIRO \& TERCEIRO 2000).

1) Desenvolvido com auxílio da FAPESP.

2) Laboratório de Morfologia de Crustáceos, Departamento de Biologia Aplicada, Universidade Estadual Paulista. Via de Acesso Prof. Paulo Donato Castellane, 14884-900 Jaboticabal, São Paulo, Brasil. E-mail: pinheiro@ fcav.unesp.br 
Os crustáceos de importância econômica preponderam sobre os demais quanto ao estudo embrionário, como é o caso dos camarões Palaemon serratus (Pennant, 1777) por CAMPILLO (1979) e RAMONELl et al. (1987), e Macrobrachium rosenbergii (De Man, 1879) por ClARKE et al. (1990); das lagostas Homarus gammarus (Linnaeus, 1758) por PANDIAN (1970a) e BRANFORD (1978) e Homarus americanus H. Milne Edwards. 1837 por PANDIAN (1970b) e ATTARD \& HUdON (1987); do caranguejo Carcinus maenas (Linnaeus, 1758) por CHEUNG (1966); e do anomuro Paralithodes platypus Brandt, 1850 por JENSEN \& ARMSTRONG (1989).

A temperatura da água mostra correlação negativa com a duração do desenvolvimento embrionário, sendo por isso considerada de grande influência durante a ontogenia (NAGAO et al. 1999). Segundo BAS \& SPIVAK (2000) a salinidade também pode atuar neste sentido, além de outros fatores intrínsecos (e.g. genéticos), que promovem alteração de tamanho nos ovos (MASHIKO 1992).

Informações sobre a duração da incubação ovariana e o desenvolvimento embrionário de $A$. cribrarius seriam de grande utilidade numa avaliação produtiva em projetos de aqüicultura, pois a sobrevivência das larvas está intimamente relacionada às suas reservas vitelínicas (GIMÉNEZ \& ANGER 2001). O potencial de cultivo dessa espécie já tem sido ressaltado na literatura, com destaque para sua fácil reprodução em cativeiro (PINHEIRO \& FRANSOZO 1999) e alta fecundidade (PINHEIRO \& TERCEIRO 2000).

O objetivo do presente trabalho é descrever os estágios embrionários do siri A. cribrarius com base na morfologia interna e coloração, analisar a biometria embrionária (diâmetro e volume do ovo), além de verificar a coerência biológica do agrupamento desses estágios. Será apresentada uma tabela sinóptica com as características peculiares a cada estágio, bem como uma revisão do assunto, enfatizando os representantes da Família Portunidae.

\section{MATERIAL E MÉTODOS}

Os exemplares de A. cribrarius foram coletados no litoral norte do Estado de São Paulo, Brasil (Ubatuba 23⒉'S), utilizando um barco camaroeiro munido de duas redes de arrasto do tipo "otter-trawl", com malha de $10 \mathrm{~mm}$ entre-nós. As amostragens foram realizadas mensalmente de agosto/1996 a julho/1997, em frente às Praias: Grande, Toninhas e Itamambuca.

As fêmeas ovígeras foram triadas e mantidas em baldes plásticos, com água do mar trocada periodicamente até a chegada ao ancoradouro. Posteriormente, foram transportadas sob aeração até o Laboratório de Morfologia de Crustáceos (Depto. de Biologia Aplicada, FCAV, UNESP Jaboticabal), onde foram mantidas em aquários marinhos com circulação fechada (filtro externo), em regime controlado de temperatura $\left(25 \pm 1^{\circ} \mathrm{C}\right)$, salinidade $(35 \pm 1 \%$ ) e fotoperíodo (12:12).

Uma pequena amostra dos ovos de cada fêmea foi examinada sob microscópio óptico, estabelecendo-se o estágio embrionário pela proporção embrião/vitelo, conforme descrito por BoOLOOTIAN et al. (1959). Ovos recém-desovados tiveram uma pequena amostra retirada a cada 24 horas e colocada em lâmina escavada para caracterização do estágio embrionário. A morfologia de cada estágio

Revta bras. Zool. 19 (2): $571-583,2002$ 
foi fotografada e esquematizada em aumento $5 x$, utilizando um microscópio óptico munido de máquina fotográfica e câmara clara. Concomitantemente, foram realizadas anotações sobre o surgimento e desenvolvimento das estruturas morfológicas diagnósticas de cada estágio, além da proporção embrião/vitelo e da coloração macroscópica do ovo.

Quinze ovos de cada estágio foram utilizados para a mensuração do diâmetro maior (DMA = funículo à margem oposta) e diâmetro menor $(\mathrm{DME}=$ entre as margens laterais, opostas ao funículo), utilizando um microscópio óptico com câmara clara. As medidas foram expressas em micrômetros $(\mu \mathrm{m})$.

A proporção DMA/DME foi estabelecida para cada um dos ovos mensurados, determinando-se a média e desvio padrão característico de cada estágio. O intervalo de confiança dessas médias foi calculado para determinar o formato do ovo $(\alpha=0,05)$ : esférico $(\mathrm{DMA} / \mathrm{DME}=1)$ ou elipsóide $(\mathrm{DMA} / \mathrm{DME} \neq 1)$. O volume dos ovos foi calculado de acordo com o formato do ovo, utilizando a equação $V=1 / 6 \pi \mathrm{d}^{3}$ se esférico (onde, $d=$ média dos dois diâmetros mensurados) ou pela equação $V=4 / 3 \pi r^{2} R$ se elipsóide (onde, $\mathrm{r}=$ raio menor $=\mathrm{DME} / 2 ; \mathrm{R}=$ raio maior $=\mathrm{DMA} / 2$ ).

As médias de diâmetro e volume de cada estágio foram submetidas a ANOVA, num delineamento inteiramente casualizado com mesmo número de repetições e confrontadas pelo teste de Tukey $(\alpha=0,05)$. Uma análise de agrupamento simples foi empregada para verificar o agrupamento entre os estágios, com base em sua similaridade morfométrica (diâmetro e volume), segundo o método descrito por ROMESBURG (1984) e KREBS (1989). A interpretação dos resultados possibilitou verificar a coerência biológica do agrupamento de estágios para $A$. cribrarius, visando facilitar sua identificação em trabalhos futuros.

\section{RESULTADOS}

Foram registrados oito estágios embrionários para A. cribrarius, a partir da exteriorização dos ovos. $\mathrm{O}$ desenvolvimento embrinário completo ocorreu em oito dias sob temperatura, salinidade e fotoperíodo controlados $\left(25 \pm 1^{\circ} \mathrm{C}, 35 \pm 1 \%\right.$ o e $12: 12$, respectivamente).

Na figura 1 encontra-se o registro fotográfico de cada um dos estágios embrionários, evidenciando suas principais características morfológicas. Baseando-se no surgimento das principais estruturas internas, coloração macroscópica e proporção embrião/vitelo, os oito estágios embrionários podem ser descritos como segue abaixo.

Estágio 1. Imediatamente após a postura, com início de clivagem (Fig. 1-A). $\mathrm{O}$ ovo apresenta seu interior totalmente preenchido por vitelo, conferindo-lhe coloração laranja escura e certa opacidade.

Estágio 2. Vitelo menos opaco. É possível diferenciar os grumos vitelínicos resultantes da clivagem (Fig. 1-B). A coloração do ovo ainda é laranja escura. Em vista lateral o embrião ocupa $1 / 8$ do ovo, sendo possível verificar estruturas ainda rudimentares por transparência.

Estágio 3. A área ocular do embrião começa a se delimitar, embora ainda não possua pigmentação (Fig. 1-C). A coloração do ovo mantem-se laranja escura, apesar do embrião ocupar 1/5 do ovo. O abdome e os maxilípedes já podem ser identificado. 

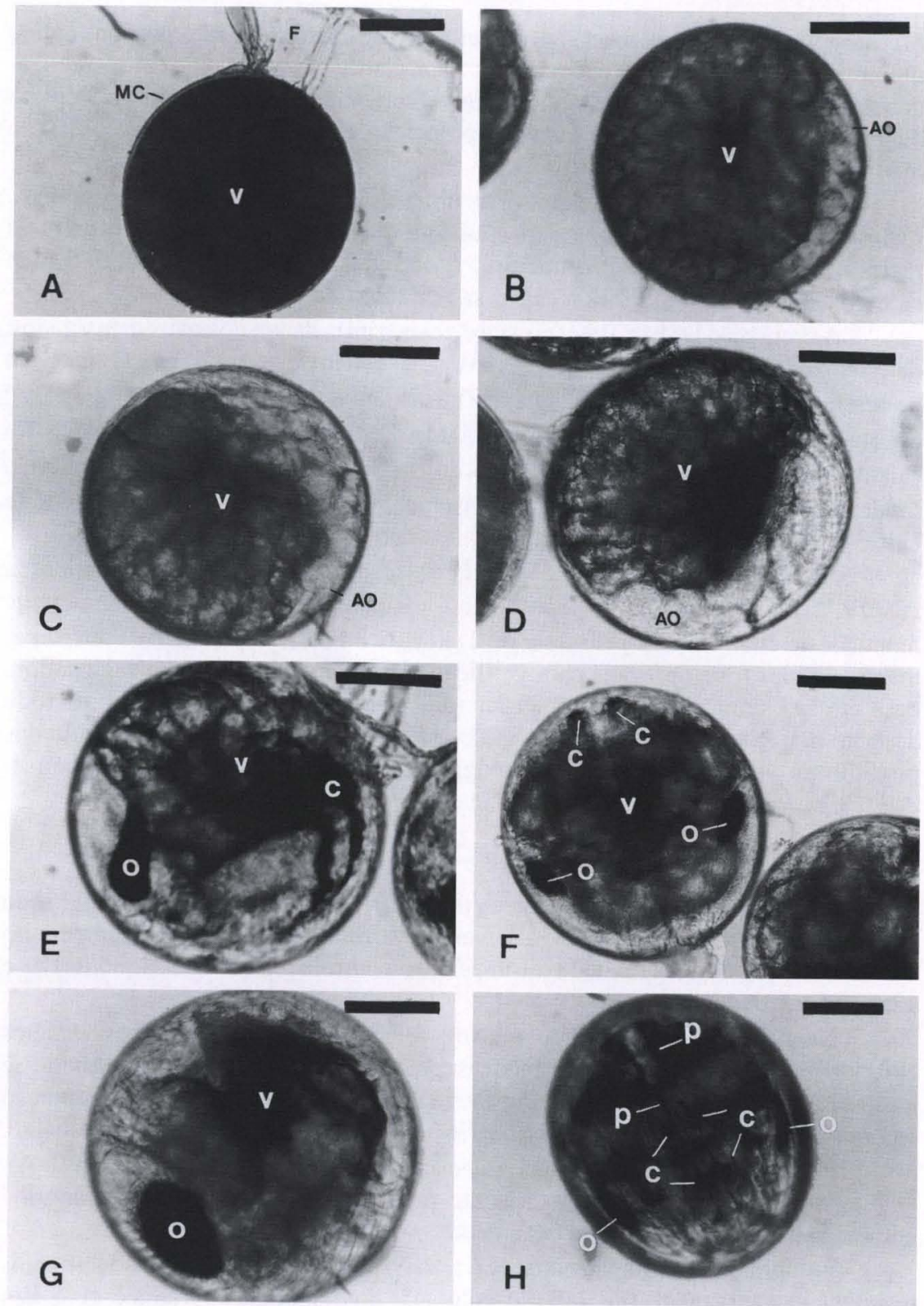

Fig. 1. Arenaeus cribrarius, registro fotográfico de cada estágio embrionário (A-D) Estágio 1-4; (E-F) Estágio 5; (G-H) Estágio 8, com destaque às estruturas morfológicas de mais fácil visualização. (AO) Área ocular, (C) cromatóforos, (F) funículo, (MC) membrana coriônica, (O) olho composto, $(P)$ ponte cromatofórica, $(V)$ vitelo. 
Estágio 4. A área ocular encontra-se bem definida (Fig. 1-D), embora uma parte ainda permaneça encoberta pelo vitelo. $\mathrm{O}$ ovo apresenta coloração ocre e o embrião ocupa $1 / 4$ do ovo. O abdome e os maxilípedes mostram-se mais alongados, com a extremidade dos últimos chegando a atingir a região ocular do embrião.

Estágio 5. Marcado pelo início da pigmentação dos olhos compostos, que apresentam formato ovalado em vista lateral (Fig. 1-E) e semilunar, em vista dorsal ou ventral. Verifica-se a formação de um par de cromatóforos no primeiro e sexto somito abdominal, facilmente visualizados quando o embrião é colocado em posição ventral (Fig. 1-F). O coração, apesar de pequeno, já pode ser identificado por seu batimento, principalmente quando o embrião é colocado em vista lateral. Início da pigmentação dos olhos e dos somitos abdominais. O embrião ocupa 1/3 do ovo, conferindo-lhe coloração marrom clara.

Estágio 6. O embrião ocupa metade do ovo, apresentando coloração marrom escura. Desenvolvimento dos cromatóforos do primeiro e sexto somitos abdominais, com início da pigmentação cromatofórica nos demais. Formação de uma ponte cromatofórica, interligando os cromatóforos abdominais ainda como um fino cordão. $\mathrm{O}$ coração do embrião cresce em tamanho, apresentando um batimento mais evidente que no estágio anterior.

Estágio 7. O coração e seus batimentos ficam mais nítidos e ocorre uma maior delimitação dos seis somitos abdominais, com diferenciação da furca do telso. O embrião ocupa $2 / 3$ do ovo, mantendo sua coloração marrom escura pelo crescimento do complexo cromatofórico e dos olhos compostos, que apresentam formato ovóide neste estágio. Em vista ventral é possível verificar um par de cromatóforos por somito abdominal, interligados pela ponte cromatofórica, que apresenta-se mais desenvolvida e dupla.

Estágio 8. A larva encontra-se totalmente formada, ocupando todo o ovo (Fig. 1-G). Em posição lateral ou dorsal é possível verificar poucos grumos vitelínicos no interior do seu cefalotórax. A coloração macroscópica do ovo é quase preta, resultado do desenvolvimento dos cromatóforos, da ponte cromatofórica do abdome (Fig. 1-H), além de um grande cromatóforo que se forma na região interna da carapaça. A carapaça, maxilípedes e somitos abdominais são evidentes e facilmente identificados por transparência.

$\mathrm{O}$ intervalo de confiança das médias para a proporção DMA/DME indica que o ovo é elipsóide durante toda a ontogenia ( $\mathrm{p}<0,05)$, com um aumento em tamanho $(24,4 \%)$ e volume $(91,9 \%)$.

Na tabela I são apresentados os dados de biometria embrionária de cada estágio. A média de diâmetro dos três primeiros estágios não mostrou diferença estatística, o que ocorreu somente a partir do quarto estágio $(\mathrm{p}<0,05)$. Os estágios 4 e 5 apresentaram média de diâmetro significativamente distinta, contrastando do sexto estágio que, por sua vez, diferiu do sétimo e oitavo, que não apresentaram contraste entre si (Fig. 2A). Fato idêntico ocorreu com as médias de volume (Fig. 2B). Nos dois casos, tornam-se perceptíveis quatro grupos, constituídos pelos estágios 1-3; 4-5; 6; e 7-8. 

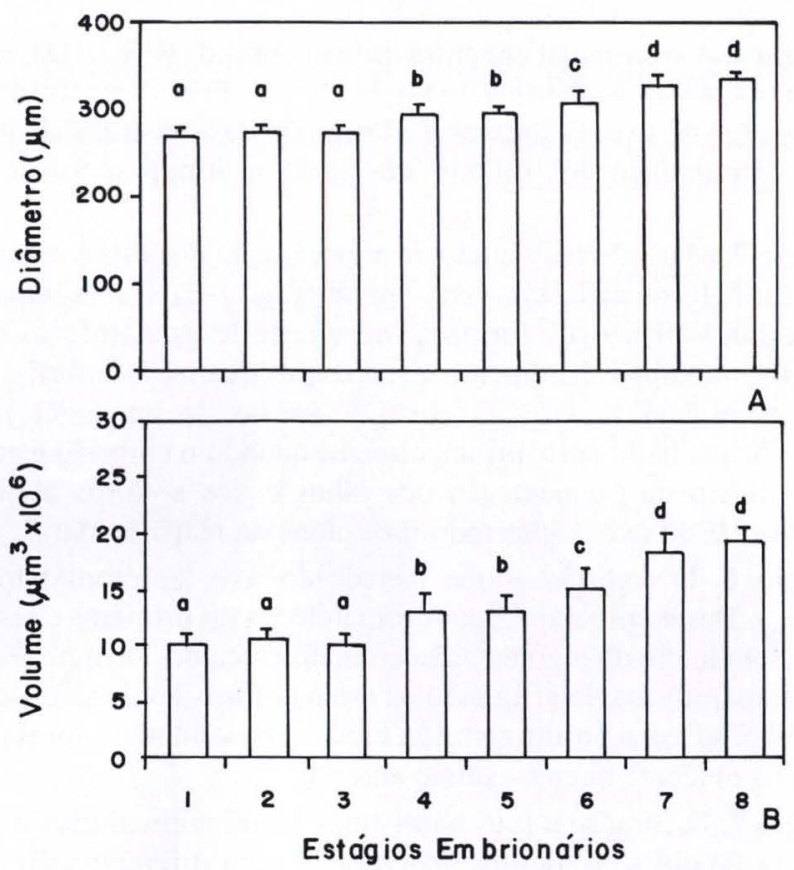

Fig. 2. Arenaeus cribrarius, médias de diâmetro (A) e de volume (B) para cada estágio embrionário e resultados da ANOVA. (Barra) Valores médios, (linha) desvio padrão. As barras associadas a uma mesma letra não apresentaram diferença significativa ( $p>0,05)$.

Tabela I. Arenaeus cribrarius, principais características do desenvolvimento embrionário da espécie.

\begin{tabular}{|c|c|c|c|c|c|c|}
\hline \multirow{2}{*}{ Estágios } & \multicolumn{3}{|c|}{ Diâmetro $(\mu \mathrm{m})$} & \multicolumn{3}{|c|}{ Volume $\left(x 10^{6} \mu \mathrm{m}^{3}\right)$} \\
\hline & Min. & Máx. & $\bar{x}_{ \pm s}$ & Min. & Máx. & $\bar{x}_{ \pm s}$ \\
\hline 1 & 252,50 & 287,50 & $269,67 \pm 9,06 a$ & 8,34 & 11,90 & $10,02 \pm 0,99 a$ \\
\hline 2 & 260,00 & 292,50 & $274,33 \pm 6,84 a$ & 8,84 & 12,99 & $10,55 \pm 0,95 a$ \\
\hline 3 & 255,00 & 290,00 & $270,50 \pm 9,26 a$ & 8,51 & 12,32 & $10,03 \pm 0,99 a$ \\
\hline 4 & 265,00 & 310,00 & $294,33 \pm 12,11 b$ & 9,56 & 15,08 & $13,07 \pm 1,58 b$ \\
\hline 5 & 275,00 & 305,00 & $295,17 \pm 8,15 b$ & 10,69 & 14,61 & $13,22 \pm 1,11 b$ \\
\hline 6 & 275,00 & 325,00 & $308,33 \pm 12,94 \mathrm{c}$ & 10,69 & 17,69 & $15,01 \pm 1,85 c$ \\
\hline 7 & 315,00 & 350,00 & $329,00 \pm 10,39 d$ & 16,10 & 21,79 & $18,29 \pm 1,68 d$ \\
\hline 8 & 325,00 & 345,00 & $335,33 \pm 6,40 d$ & 17,11 & 21,18 & $19,28 \pm 1,17 d$ \\
\hline
\end{tabular}

*As médias em uma mesma coluna, associadas a uma mesma letra minúscula, não mostraram diferença estatística $(p>0,05)$.

A análise de agrupamento revelou a existência de três grupos (1-3; 4-6; 7-8), independente da variável biométrica, considerando a dissimilaridade biométrica na distância euclidiana $80 \mu \mathrm{m}$ (Fig. 3A) para o diâmetro e $11 \times 10^{6} \mu \mathrm{m}^{3}$ (Fig. 3B) para o volume. 

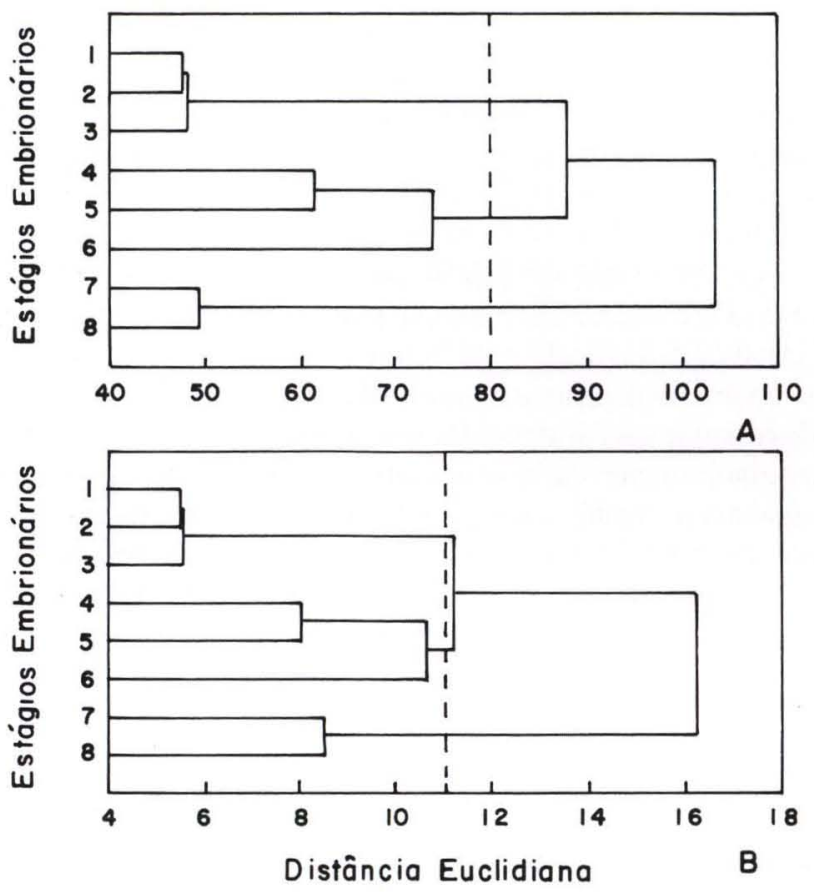

Fig. 3. Arenaeus cribrarius, cladogramas da análise de agrupamento simples dos estágios embrionários com base no diâmetro $(A)$ e no volume $(B)$.

\section{DISCUSSÃO}

Segundo KAESTNER (1970) o padrão de desenvolvimento dos crustáceos é extremamente diversificado. Em algumas espécies a progressão metamérica ocorre integralmente dentro do ovo e o animal eclode com forma similar à do adulto (desenvolvimento epimórfico), enquanto em outras só ocorre pós-embrionariamente, após passar por fases larvais peculiares (desenvolvimento anamórfico). De acordo com STUCK \& TRUSDALE (1988), A. cribrarius passa por oito estágios de zoea e um de megalopa, o que caracteriza um desenvolvimento do tipo anamórfico irregular, como ocorre com outros braquiúros marinhos.

O desenvolvimento embrionário dos crustáceos pode ser dividido didaticamente em estágios, identificados pela modificação e/ou surgimento de certas estruturas morfológicas e alterações biométricas durante a ontogenia. O registro de oito estágios embrionários para $A$. cribrarius, diferiu numericamente dos 10 estabelecidos por BOOLOOTIAN et al. (1959) e dos 11 mencionados por BROEKHUYSEN (1936). No entanto, BOOLOOTIAN et al. (1959) caracterizaram o décimo estágio pelo rompimento da membrana coriônica e liberação da larva, o que é de difícil constatação em trabalhos biológicos, devido à rapidez deste evento. Além disso, sabe-se que a temperatura apresenta correlação negativa com a duração do desenvolvimento embrionário (EFFORD 1969; WEAR 1974; FUKUI 1988; FUROTA 1988) e larval nos crustáceos decápodos (SASTRY 1983; LINDLEY 1990a,b; PINHEIRO et al. 1994). 
A mudança de coloração dos ovos durante a ontogenia é uma característica muito comum nos crustáceos. As alterações cromáticas variam interespecificamente, p. ex. laranja para marrom escuro nos portunídeos Callinectes ornatus Ordway, 1863 e C. danae Smith, 1869, de acordo com MANTEllatTo \& FRANSOZO (1997) e COSTA \& NEGREIROS-FRANSOZO (1996), respectivamente. O mesmo ocorreu com A._cribrarius, caracterizando um padrão para a Família Portunidae.

Segundo GHIDALIA (1985) os carotenos são hidrocarbonetos que formam complexos carotenóides quando combinados com proteínas ou lipoproteínas, sendo encontrados em diferentes partes do corpo dos crustáceos. Na revisão desse autor, o acúmulo dos complexos carotenóides nos ovos e ovários, bem como seu consumo durante o desenvolvimento embrionário ainda é questionável, embora sua concentração decresça inversamente, em alguns decápodos, com o crescimento do embrião. Os pigmentos carotenóides também atuam na absorção de radiações do espectro solar, principalmente a ultravioleta, conferindo proteção ao embrião de seus efeitos nocivos (GREEN 1957). Certamente o hábito bentônico de $A$. cribrarius faz com que esta função seja minimizada, já que a coluna d'água filtraria essa radiação, extremamente prejudicial a divisão celular e embriogênese. Além disso, GREEN (1957) trabalhou com Daphinia onde os embriões ficam muito mais expostos.

O aumento de diâmetro e volume dos ovos durante o desenvolvimento é um padrão para os crustáceos, estando associado às mudanças na forma do ovo. $\mathrm{O}$ percentual de aumento do ovo varia interespecificamente nos crustáceos decápodos (Tab. II), mesmo quando esta comparação é feita entre espécies de uma mesma família. Os ovos de A. cribrarius são levemente elípticos durante todo o desenvolvimento. GIMÉNEZ \& ANGER (2001) verificaram que fêmeas de uma mesma espécie, submetidas a condições ambientais idênticas apresentaram variação no tamanho dos ovos, indicando a existência de fatores intrínsecos que regulam estas alterações biométricas. A temperatura (WEAR 1974, STEELE \& STEELE 1975) e salinidade (BAS \& SPIVAK 2000) são os fatores abióticos considerados de maior relevância para a variabilidade de tamanho dos ovos. A idade e tamanho das fêmeas (STELla et al. 1996), bem como fatores genéticos (MASHIKO 1992), podem também promover alterações ontogenéticas de tamanho. Segundo BAS \& SPIVAK (2000), a formação incompleta da membrana coriônica nos primeiros estágios embrionários é considerada responsável pela maior suscetibilidade às alterações de salinidade, o que explicaria a pequena variação do tamanho dos ovos, nos estágios iniciais (1-5). O fenômeno de osmose é responsável pelo rompimento da membrana coriônica no estágio final, facilitando a liberação da larva no momento da eclosão (DAVIS 1964; WEAR 1974).

Os ovos de A. cribrarius são de pequeno porte $(270$ a $335 \mu \mathrm{m})$, muito similares aos de Callinectes sapidus Rathbun, 1876 (273 a $320 \mu \mathrm{m}$ ), segundo DAVIS (1965), e de Ovalipes punctatus (De Haan, 1833) (347 a $378 \mu \mathrm{m})$, conforme DU PREEZ \& MCLACHLAN (1984). Os crustáceos decápodos que apresentam ovos pequenos possuem um desenvolvimento embrionário mais rápido, ocorrendo o inverso com aqueles que apresentam ovos maiores (HERRING 1974). Por outro lado, a menor quantidade de energia vitelínica nos ovos de menor porte faz com que a prole passe por um maior número de estágios larvais e permaneça por mais tempo 
no plâncton, quando estão mais suscetíveis à predação (LINDLEY 1990a,b; PINHEIRO et al. 1994). O meio ambiente também modula o tempo de desenvolvimento dos ovos (FUROTA 1988; FUKUI 1988), enquanto fatores de ordem fisiológica são os principais responsáveis por variações biométricas do ovo em alguns crustáceos (CRISP \& COSTlow 1963; EFFORD 1969; Nishino 1980). Segundo GimÉnEZ \& ANGER (2001) a idade das fêmeas também é um dos fatores responsáveis pela variação no tamanho e volume dos ovos.

Tabela II. Revisão sobre o percentual de aumento dos ovos em diâmetro e volume durante a ontogenia e o número de estágios embrionários das espécies da infraordem Brachyura analisadas até o momento.

\begin{tabular}{|c|c|c|c|c|c|}
\hline Espécie & Autor & Local & $\begin{array}{c}\text { Diâmetro* } \\
(\%)\end{array}$ & $\begin{array}{c}\text { Volume** } \\
(\%)\end{array}$ & $\begin{array}{c}\text { Número de } \\
\text { estágios }\end{array}$ \\
\hline \multicolumn{6}{|l|}{ Portunidae } \\
\hline \multirow[t]{2}{*}{ Arenaeus cribrarius } & Pinheiro \& Terceiro (2000) & Ubatuba, Brasil & - & - & 3 \\
\hline & Presente estudo & Ubatuba, Brasil & 24,4 & 91,9 & 8 \\
\hline Callinectes sapidus & Millikin \& Williams (1984) & EUA & 17,2 & - & - \\
\hline Callinectes danae & Costa \& Negreiros-Fransozo (1996) & Ubatuba, Brasil & - & - & 3 \\
\hline \multirow[t]{2}{*}{ Carcinus maenas } & Broekhuysen (1936) & Den Helder, Holanda & - & - & 11 \\
\hline & Wear (1974) & Hong kong, China & - & 80,0 & - \\
\hline Charybdis (C.) feriatus & Campbell \& Fielder (1988) & Queensland, Austrália & - & 24,9 & 3 \\
\hline Macropipus depurador & Wear (1974) & Hong Kong, China & - & 120,0 & - \\
\hline Macropipus pusillus & Wear (1974) & Hong Kong, China & - & 130,0 & - \\
\hline Necora puber & Valdes et al. (1991) & La Coruña, Espanha & 22,0 & 88,4 & 5 \\
\hline \multirow[t]{2}{*}{ Ovalipes catharus } & Haddon (1994) & Nova Zelândia & 29,3 & - & 5 \\
\hline & Du Preez \& McLachlan (1984) & Port Elizabeth, África do Sul & 8,9 & - & 3 \\
\hline \multirow[t]{2}{*}{ Portunus pelagicus } & Batoy et al. (1987) & Bohol, Filipinas & 40,0 & - & 6 \\
\hline & Campbell \& Fielder (1988) & Queensland, Austrália & - & 141,5 & 3 \\
\hline Portunus sanguinolentus & Campbell \& Fielder (1988) & Queensland, Austrália & - & 89,7 & 3 \\
\hline Portunus spinimanus & Santos \& Negreiros-Fransozo (1998) & Ubatuba, Brasil & - & - & 3 \\
\hline \multicolumn{6}{|l|}{ Majidae } \\
\hline Eurynome aspersa & Wear (1974) & Hong Kong, China & - & 95,0 & - \\
\hline Inachus dorsettensis & Wear (1974) & Hong Kong, China & - & 85,0 & - \\
\hline Macropodia longirostris & Wear (1974) & Hong Kong, China & - & 135,0 & - \\
\hline Macropodia rostrata & Wear (1974) & Hong Kong, China & - & 120,0 & - \\
\hline Maia squinado & Wear (1974) & Hong Kong, China & - & 65,0 & - \\
\hline \multicolumn{6}{|l|}{ Leucosiidae } \\
\hline Ebalia tuberosa & Wear (1974) & Hong Kong, China & - & 120,0 & - \\
\hline \multicolumn{6}{|l|}{ Goneplacidae } \\
\hline Goneplax rhomboides & Wear (1974) & Hong Kong, China & - & 120,0 & - \\
\hline \multicolumn{6}{|l|}{ Grapsidae } \\
\hline Hemigrapsus nudus & Boolootian et al. (1959) & Califórnia, EUA & - & - & 10 \\
\hline Pachygrapsus crassipes & Boolootian et al. (1959) & Califórnia, EUA & - & - & 10 \\
\hline \multicolumn{6}{|l|}{ Cancridae } \\
\hline Cancer pagurus & Wear (1974) & Hong kong, China & - & 50,0 & - \\
\hline \multicolumn{6}{|l|}{ Atelecyclidae } \\
\hline \multirow[t]{2}{*}{ Erimacrus isenbeckii } & Nagao et al. (1999) & Hokkaido, Japão & 11,1 & - & 9 \\
\hline & Yoshida (1940) & Coréia do Norte & - & - & 3 \\
\hline
\end{tabular}

*) Percentual de aumento em diâmetro da postura até a eclosão; **) Percentual de aumento do volume da postura até a eclosão. 
O número de estágios embrionários estabelecidos pelos carcinólogos varia bastante entre as diferentes espécies de braquiúros (Tab. II). Necora puber (Linnaeus, 1767) apresentou cinco estágios (VALDES et al. 1991), sendo os dois primeiros similares aos de A. cribrarius, enquanto os demais são idênticos aos estágios 5 a 8 . NAGAO et al. (1999) estabeleceu nove estágios embrionários para Erimacrus isenbeckii (Brandt, 1848) enquanto YosHIDA (1940) menciona três para a mesma espécie, embora não defina claramente suas características. GIMÉNEZ \& ANGER (2001) determinaram nove estágios para Chasmagnathus granulata, sendo o oitavo e nono similares ao último estágio de $A$. cribrarius. As análises estatísticas revelam a possibilidade de agrupamento dos oito estágios embrionários de $A$. cribrarius em três grupos, corroborando os dados de PINHEIRO \& TERCEIRO (2000), que estabeleceram os estágios pela proporção embrião/vitelo e surgimento/desenvolvimento dos olhos compostos e cromatóforos, a saber: inicial (1-3), intermediário (4-6) e final: (7-8). Esta divisão didática, com fundamentação biológica/biométrica tem sido seguida por outros autores que trabalharam com portunídeos, como COSTA \& NEgreiros-Fransozo (1996), Mantelatto \& Fransozo (1997) e SANTOS \& NEGREIROS-FRANSOZO (1998).

A identificação dos estágios embrionários de A. cribrarius com base apenas na morfometria e cromatologia é extremamente precária, mas torna eficiente quando combinada com a determinação da proporção embrião/vitelo e surgimento/desenvolvimento das principais estruturas internas (e.g. olhos compostos e cromatóforos). O estabelecimento de três estágios (inicial, intermediário e final) pode facilitar a interpretação biológica e a aplicação de análises estatísticas em estudos de biologia reprodutiva, bem como uma delimitação mais fiel da época de desova de uma espécie.

AGRADECIMENTOS. À FAPESP pelo financiamento do Projeto Arenaeus (Proc. 95/9495-2) e concessão de um veículo para as coletas de campo (Proc. 94/4878-8). Ao Dr. Carlos F. Damião Filho e Dra. Fabíola Vitti Moro (Depto de Biologia Aplicada, FCAV, UNESP, Jaboticabal) pelo uso do microscópio óptico com sistema fotográfico e auxílio na revelação fotográfica. À bióloga Nancy Prette-Varandas pelo auxílio no processamento e análise dos dados.

\section{REFERÊNCIAS BIBLIOGRÁFICAS}

Anderson, D.T. 1982. Embriology, p. 1-41. In: L.G. Abele (Ed.). The Biology of Crustacea. Embriology, Morphology, and Genetics. New York, Academic Press, II+440p.

ATtARD, J. \& C. HudON. 1987. Embryonic development and energetic investment in egg production in relation to size of female lobster (Homarus_americanus). Canadian Jour. Fish. Aquat. Sci. 44: $1157-1164$.

Bas, C.C. \& E.D. SpIvaK. 2000. Effect of salinity on embryos of two southwestern atlantic estuarine grapsidae crab species culture in vitro. Jour. Crust. Biol. 20 (4): 647-656.

Batoy, C.B.; J.F. Samargo \& B.C. PILAPIL. 1987. Breeding season, sexual maturity and fecundity of the blue crab, Portunus pelagicus (L.) in selected coastal waters in Leyte and vicinity, Philippines. Ann. Trop. Res. 9: 157-177.

Boolootian, R.A.; A.C. Giese; A. Farmanfarmaian \& J. Tucker. 1959. Reproductive cycles of five west coast crabs. Physiol. Zool. 32: 213-220.

BRANFORD, J.R, 1978. Incubation period for the lobster Homarus gammarus at various temperatures. Mar. Biol. Berlin. 47: 363-368. 
BroekHuYSEn JR., G.J. 1936. On development, growth and distribution of Carcinides maenas (L.). Arch. Neerl. Zool. 2: 257-399.

CAmpbell, G.R. \& D.R. Fielder. 1988. Egg extrusion and egg development in three species of commercially important portunidae crabs from S.E. Queensland. Proc. R. Soc. Queensl. 99: 93-100.

CAmpillo, A. 1979. Contribution à l'etude de la crevette rose Palaemon serratus (Pennant). Exploitation, biologie. Rev. Trav. Inst. Pêches Marit. 43: 293-352.

Cheung, T.S. 1966. The development of egg membranes and egg attachment in the shore crab, Carcinus maenas and some related decapods. Jour. Mar. Biol. Assoc. U.K. 46: 373-400.

Clarke, A.; J.H. Brown \& L.J. Holmes.1990. The biochemical composition of eggs from Macrobrachium rosenbergii in relation to embryonic development. Comp. Biochem. Physiol. 96 (3): 505-511.

Costa, T.M. \& M.L. Negreiros-Fransozo. 1996. Fecundidade de Callinectes danae Smith,1869 (Crustacea, Decapoda, Portunidae) na região de Ubatuba (SP), Brasil. Arq. Biol. Tecnol., Curitiba, 39 (2): 393-400.

CRISP, D. \& J. Costlow. 1963. The tolerance of developing cirripede embryos to salinity and temperature. Oikos 14: 22-34.

DAvIS, C.C. 1964. A study of the hatching process in aquatic invertebrates. IX. Hatching with the brood sac of oviviparous isopod, Cirolana sp. (Isopoda, Cirolanidae). IX. Hatching in the freshwater shrimps, Potimirim glabra (Kingsley) (Macrura, Atyidae). Pacif. Sci. 18 (4): 378-384.

-1965. A study of the hatching process in aquatic invertebrates: XX. The blue crab Callinectes sapidus Rathbun. XXI. The nemertean Carcinonemertes carcinophila (Kölliker). Chesapeake Sci. 6: 201-208.

Du Preez, H.H. \& A. McLachlan. 1984. Biology of the three spot swimming crab, Ovalipes punctatus (De Haan) III. Reproduction, fecundity and egg development. Crustaceana 47 (3): 285-297.

EFFORD, I.E. 1969. Egg size in the sand crab, Emerita analoga (Decapoda, Hippidae). Crustaceana 16 (2): $15-26$.

Fukui, Y. 1988. Comparative studies on the life history of the grapsid crabs (Crustacea, Brachyura) inhabiting intertidal cobble and boulder shores. Publ. Seto Mar. Biol. Lab. 33: 121-162.

Furota, T. 1988. The ecology of introduced spider crab Pyromaia tuberculata in an organically polluted bay (Preliminary Report). Benthos Res. 33/34: 79-89.

GHIDALIA, W. 1985. Structural and biological aspects of pigments, p. 301-375. In: D.E. BLISS \& L.H. Mantel (Eds). The Biology of Crustacea. Integument, Pigments, and Hormonal Processes. New York, Academic Press, IX+550p.

GimÉNEZ, L. \& K. ANGER. 2001.Relationships among salinity, egg size, embryonic development, and larval biomass in the estuarine crab Chasmagnathus granulata Dana, (1851). Jour. Exp. Mar. Biol. Ecol. 260: 241-257.

Green, J. 1957. Carotenoids in Daphnia., Proc. R. Soc. London Serv. B. 147: $392-401$.

HADDON, M. 1994. Size-fecundity relationships, mating behaviour and larval release in the New Zealand paddle crab Ovalipes catharus (White, 1843) (Brachyura: Portunidae). N.Z Jour. Mar. Freshwater Res. 28: 329-334.

HERRING, P.J. 1974. Observations on the embryonic development of some deep-living decapod crustaceans, with particular reference to species of Acanthephyra. Mar. Biol. Berlin 25 (1): 25-34.

Jensen, G.C. \& D.A. Armstrong. 1989. Biennial reproductive cycle of blue king crab, Paralithodes platypus at the Pribiolof Islands, Alaska, and comparison to a congener, $P$. camtchatica. Canadian Jour. Fish. Aquat. Sci. 46: 932-940.

KAESTNER, A. 1970. Invertebrate Zoology. New York, Interscience Publishers, III+523p.

KREBS, J.C. 1989. Cluster Analysis, p. 310-327. In: S. PISANo (Ed.). Ecological Methodology. New York, Haper \& Row, 654p.

Lindley, J.A. 1990a. Regressions for estimating development in the shrimp, Palaemonetes pugio Holthuis (Caridea, Palaemonidae). Crustaceana (Suppl. 2): 19-26. 
$1990 \mathrm{~b}$. Regressions for estimating development times of the pelagic larvae of Paguridae and Porcellanidae. Jour. Plankton Res. 12 (3): 673-678.

Mantellato, F.L.M. \& A. Fransozo. 1997. Fecundity of the crab Callinectes ornatus Ordway, 1863 (Decapoda, Brachyura, Portunidae) from the Ubatuba region, São Paulo, Brazil. Crustaceana 70 (2): 214-226.

MASHIKo, K. 1992. Genetic egg and clutch size variations in freshwater prawn populations. Oikos 63: 454-458.

Millikin, M.R. \& A.B. Williams. 1984. Synopsis of Biological data on the crab, Callinectes sapidus Rathbun. NOAA. Tech. Rep. NMFS. 1 (138): 1-39.

NAGAO, J.; H. MunehaRA \& K. ShimaZAKI. 1999. Embryonic development of the hair crab Erimacrus isenbeckii. Jour. Crust. Biol. 19 (1): 77-83.

Nishino, M. 1980. Geographical variation in body size, brood size and egg size of a freshwater shrimp, Palaemon paucidens De Haan, with some discussion on brood habit. Japan. Jour. Limnol. 41: 185-202.

Pandian, T.J. 1970a. Ecophysiological studies on the developing eggs and embryos of the European lobster Homarus gammarus. Mar. Biol. Berlin 5: 154-167.

. 1970b. Yolk utilization and hatching in the Canadian lobster Homarus americanus. Mar. Biol. Berlin 7: 249-254.

Pinheiro, M.A.A. \& A. Fransozo. 1998. Sexual Maturity of the speckled swimming crab Arenaeus cribrarius (Lamarck, 1818) (Crustacea, Brachyura: Portunidae) in Ubatuba Litoral, São Paulo State, Brazil. Crustaceana 71 (4): 434-452.

- 1999. Reproductive behavior of the swimming crab Arenaeus cribrarius (Lamarck, 1818) (Crustacea, Brachyura, Portunidae) in captivity. Bull. Mar. Sci. 64 (2): 243-253.

- 2002. Reproductive dynamics of the speckled swimming crab Arenaeus cribrarius (Lamarck, 1818) (Brachyura, Portunidae), on the north coast of São Paulo State, Brazil. Jour. Crust. Biol. 22 (2): 416-428.

Pinheiro, M.A.A. \& O.S.L. Terceiro. 2000. Fecundity and reproductive output of the speckled swimming crab, Arenaeus cribrarius (Lamarck, 1818) (Brachyura, Portunidae). Crustaceana 73 (9): 1121-1137.

Pinheiro, M.A.A.; A. Fransozo \& M.L. Negreiros-Fransozo. 1994. Estimativa da duração larval em função da temperatura para a Família Majidae (Crustacea, Decapoda, Brachyura). Bol. Inst. Pesca, São Paulo, 21: 75-81.

Ramonell, R.; R. Bermudez \& A. Landim. 1987. Maduración de los huevos de Palaemon serratus (Pennant) e influencia de la temperatura en tiempo de incubación. Cuad. Marisq. Publ. Tec. 12: 285-290.

RomesbuRG, H.C. 1984. Cluster Analysis for Researchers. Belmont, Lifetime Learning Publications, $330 \mathrm{p}$.

SAINTE-MARIE, B. 1993. Reproductive cycle and fecundity of primiparous and multiparous female snow crab, in the northwest Gulf of Saint Lawrence. Can. Jour. Fish. Aquat. Sci. 50 (10): 2147-2156.

Santos, S. \& M.L Negreiros-Fransozo. 1998. Fecundity in Portunus spinimanus Latreille, 1819 (Crustacea, Brachyura, Portunidae) from Ubatuba, São Paulo, Brazil. Interciencia 22 (5): 259-263.

SASTRY, A.N. 1983. Ecological aspects of reproduction, p. 179-270. In: F.J. VERNBERG \& W.B. VERnBERg (Eds.). The Biology of Crustacea. Environmental Adaptations. New York, Academic Press, V+471p.

STEele, D. \& V. STEele. 1975. Egg size and duration of embryonic development in Crustacea. Int. Rev. Gesamten Hydrobiol. 60: 711-715.

STElla, V.; L. LóPEZ \& E. RodríGuez. 1996. Fecundity and brood biomass investment in the estuarine crab Chasmagnathus granulata Dana, 1851. Crustaceana 69: 307-312.

STUCK, K.C. \& F.M. TRUeSDALE. 1988. Larval development of the speckled swimming crab, Arenaeus cribrarius (Decapoda: Brachyura: Portunidae) reared in the laboratory. Bull. Mar. Sci. 42 (1): 101-132. 
VAldes, L.; M.T. Alvarez-Ossorio \& E. GonZÁlez-GurRiarán. 1991. Incubation of eggs of Necora puber (L., 1767) (Decapoda, Brachyura, Portunidae). Volume and biomass changes in embryonic development. Crustaceana 60 (2): 163-177.

WEAR, R.G. 1974. Incubation in British Decapod Crustacea, and the effects of temperature on the rate and success of embryonic development. Jour. Mar. Biol. Ass. U.K. 54: 745-762.

YosHIDA, Y. 1940. On the reproduction of useful crab species from North Korea. Suisan kenkyushi 35: 4-12.

Recebido em 19.X.2001; aceito em 17.V.2002. 